\title{
MODELOS ACOPLADOS DO IPCC-AR4 E O GRADIENTE MERIDIONAL DE TEMPERATURA DA SUPERFÍCIE DO MAR NO ATLÂNTICO TROPICAL: RELAÇÕES COM A PRECIPITAÇÃO NO NORTE DO NORDESTE DO BRASIL
}

\author{
JOSÉ MARIA BRABO ALVES ${ }^{1}$, ROSANE RODRIGUES CHAVES ${ }^{2}$, JACQUES SERVAIN ${ }^{3,4}$, \\ EVERALDO BARREIROS DE SOUZA ${ }^{5}$, EMERSON MARIANO DA SILVA ${ }^{1}$, ANTONONIO CARLOS \\ SANTANA DOS SANTOS ${ }^{1}$, AUGUSTO CÉSAR BARROS BARBOSA ${ }^{1}$, CARLOS JACINTO \\ DE OLIVEIRA ${ }^{1}$, SÉRGIO SOUSA SOMBRA ${ }^{1}$, ALEXANDRE ARAÚJO COSTA $^{1}$, FRANCISCO \\ GERALDO DE MELO PINHEIRO ${ }^{1}$
}
${ }^{1}$ Universidade Estadual do Ceará (UECE), Departamento de Física, Centro de Ciência e Tecnologia (CCT), Fortaleza, CE, Brasil
${ }^{2}$ Universidade Federal do Rio Grande do Norte (UFRN), Natal, RN, Brasil
${ }^{3}$ Institut de Recherche pour Le Développement (IRD), Paris, France
${ }^{4}$ Fundação Cearense de Meteorologia e Recursos Hídricos (FUNCEME), Fortaleza, CE, Brasil
${ }^{5}$ Universidade Federal do Pará, Instituto de Geociências, Faculdade de Meteorologia, Belém, PA, Brasil

braboalves@gmail.com

Recebido Fevereiro de 2013 - Aceito Dezembro de 2013

\begin{abstract}
RESUMO
Este artigo mostra como três modelos acoplados do Intergovernmental on Panel Climate Change - (IPCC-AR4), o FGOALS1.0G - LASG do Institute of Atmospheric Physics of China, o GISSER da National Aeronautics Space Admnistration (NASA) e o GFDL_CM2 da National Oceanic and Atmospheric Administration (NOAA), simularam a variabilidade do gradiente meridional de Temperatura da Superfície do Mar (TSM), entre os meses de fevereiro a maio, no Atlântico Tropical (1901-1999). A precipitação durante a estação chuvosa (fevereiro a maio) no setor norte do Nordeste do Brasil (NEB) foi também analisada pelos três modelos e comparada com as observações. Os modelos GISSER e FGOALS1.0G mostraram melhor desempenho na simulação do sinal do gradiente meridional de TSM no Atlântico Tropical para o período de 1901 a 1999. Destaca-se que os modelos apresentaram um melhor desempenho na simulação da tendência decadal, conseguindo explicar entre $50 \%$ a $80 \%$ da variabilidade do gradiente, com a TSM do setor sul sendo melhor simulada.

Palavras-chave: Oceano Atlântico Tropical, simulação-previsão, variabilidade/mudança climática.
\end{abstract}

ABSTRACT: IPCC-AR4 COUPLED MODELS AND THE MERIDIONAL GRADIENT OF SEA SURFACE TEMPERATURE IN THE TROPICAL ATLANTIC: RELATIONSHIP WITH THE PRECIPITATION OVER THE NORTHER NORTHEAST OF BRAZIL

This article shows how three coupled models of the Intergovernmental Panel on Climate ChangeFourth Assessment Report (IPCC-AR4), the FGOALS1.0G - LASG of the Institute of Atmospheric Physics of China, the GISSER of the National Aeronautics and Space Administration (NASA) and the GFDL_CM2 of the National Oceanic and Atmospheric Administration (NOAA) have simulated the meridional gradient variability of the Sea Surface Temperature (SST) in the tropical Atlantic during February to May months for the period from 1901 to 1999. The precipitation during the rainy season (February-May) in the northern sector of Northeast Brazil (NEB) was also analyzed by those three models and compared with observations. The GISSER and FGOALS1.0G models showed better performance at signal simulation of signal of the meridional SST gradient over the tropical Atlantic for the period 1901-1999. It is noteworthy that the coupled models have performed better 
in simulating the decadal trend, explaining $50 \%$ to $80 \%$ of gradient variability, being the southern sector SST better simulated.

Keywords: Tropical Atlantic Ocean, simulation-forecast, climate variability/change.

\section{INTRODUÇÃO}

Os oceanos cobrem mais de $72 \%$ do planeta, e suas variações térmicas são responsáveis por diversos eventos meteorológicos e climáticos em várias regiões do globo. A simulação e a previsão das variações térmicas no Atlântico Tropical, com ênfase no gradiente meridional de temperatura de superfície do mar (TSM) são imprescindíveis para o aprimoramento das previsões sazonais de precipitação no Nordeste do Brasil (NEB). O Oceano Atlântico Tropical tem uma complexa variabilidade física intrassazonal, anual e interanual com alguns modos de variabilidade térmicos principais que definem a chamada Variabilidade do Atlântico Tropical (TAV - Tropical Atlantic Variability, Tanimoto e Xie, 2002). Entre estes modos merecem destaque devido à maior influência no clima das Américas e África, e que ocorrem na faixa tropical do Atlântico, o modo equatorial, chamado de "El Niño do Atlântico" (Zebiak, 1993) e o modo denominado de dipolo ou de gradiente inter-hemisférico (Servain e Legler, 1986 e Nobre e Shukla, 1996, entre outros).

Alguns estudos de modelos acoplados oceano-atmosfera do IPCC-AR4 tem investigado a simulação de suas anomalias de TSMs em relação ao gradiente meridional de TSM no Atlântico Tropical (Breugem et al. 2006 e Good et al. 2009).

Devido à relevada importância do gradiente meridional de TSM no Atlântico Tropical para o clima do Nordeste do Brasil (Figura 1) e parte Oeste da África em particular, o presente estudo tem como objetivo avançar no conhecimento científico deste tema. Principalmente, porque estes modelos do IPCC-AR4 são usados para se inferir projeções do clima futuro.

\section{DADOS USADOS E METODOLOGIA}

\subsection{Dados observados}

Para o período de 1901-1999 foram usados dados de TSM denominados Optimum Interpolation cujos detalhes são dados em Smith et al. (2008). Estes dados estão em pontos de grade de $1,0^{\circ}$ x $1,0^{\circ}$ de latitude e longitude sobre todos os oceanos do globo. Dados de vento das componentes meridional e zonal em $1000 \mathrm{hPa}$ e precipitação das reanálises (Kalnay et al. 1996) do National Center Environment Prediction - National and Ocean Atnospheric Admistration (NCEP/ NOAA) dos Estados Unidos também foram usados como base de dados observados para comparar com as simulações dos modelos. Além disso, utilizaram-se os dados de TSM (período de 1950-1993) para calcular correlação linear na bacia do Oceano Atlântico com um índice de precipitação observada e simulada para o setor norte do NEB definido a seguir, e fluxo de calor latente do Tropical do Comphreensive Ocean data Sets - COADS (da Silva et al., 1994). Para os dados de precipitação (1901-1999) foram usados os da Universidade de East Anglia - Climate Research Unit (CRU). Detalhes dessa base de dados que estão em uma grade de $0,5^{\circ}$ versus $0,5^{\circ}$ de latitude e longitude globais são encontrados em New et al. 1999, 2000. Com esses dados foi calculado um índice regional de precipitação para o setor norte do $\mathrm{NEB}\left(2^{\circ} \mathrm{S}-12^{\circ} \mathrm{S}\right.$ e $45^{\circ} \mathrm{W}-37^{\circ} \mathrm{W}$ - Figura 1 ).

\subsection{Dados modelados}

Para os dados do IPCC-AR4, dados mensais de TSM usados foram os dos modelos GISSER (cinco rodadas), FGOALS1.0G (duas rodadas) e o GFDL_CM2.0 (três rodadas), e para precipitação o número de rodadas foi GISSER (oito rodadas), FGOALS1.0G (três rodadas) e o GFDL_CM2.0 (três rodadas). Alguns detalhes sobre as características destes modelos acoplados oceano-atmosfera (resolução espacial horizontal e vertical na atmosfera e oceano), podem ser visto na Tabela 1. Quanto a informação sobre o volume de gases de efeito estufa nas rodadas entre 1880-1999 podem ser encontradas em http:www-pcmdi.llnl.gov/. Em geral, as rodadas

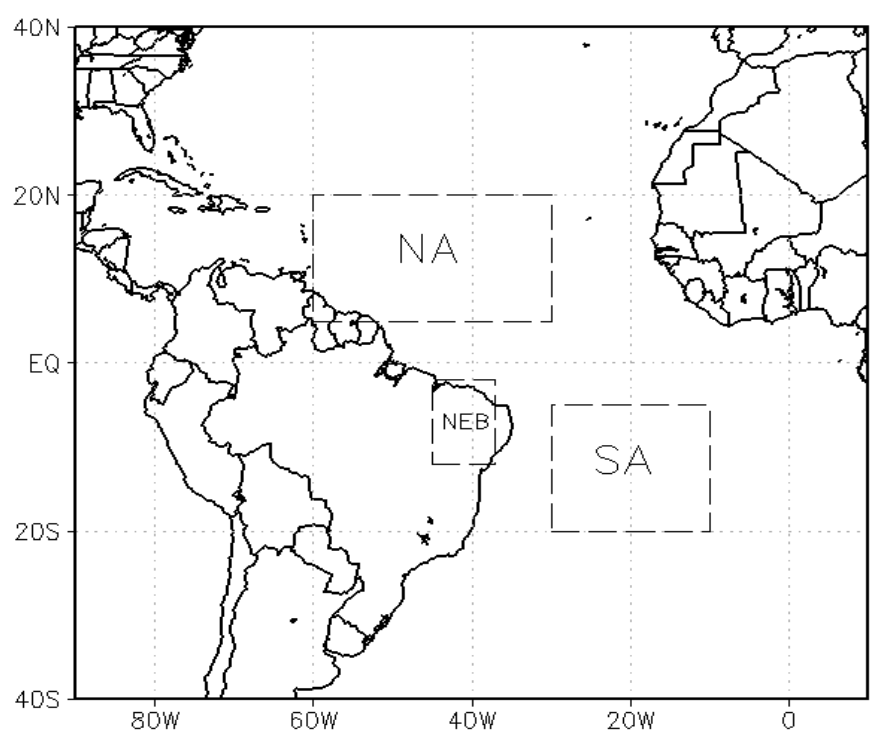

Figura 1 - Áreas norte (NA) e sul (SA) do gradiente meridional de TSM no Atlântico Tropical e setor norte do Nordeste do Brasil (NEB). 
Tabela 1 - Algumas características dos modelos do IPCC-AR4 usados no estudo e referências sobre os mesmos.

\begin{tabular}{|c|c|c|}
\hline Modelo & Resolução/topo atmosfera & Referência Básica \\
\hline FGOALS1.0G 1.0 & $64 \times 72-\mathrm{L} 32\left(2,8^{\circ}\right.$ Lat $\times 2,8^{\circ}$ Lon $)-2 \mathrm{hPa}$ & Zang e MaCFarlane (1995) \\
\hline GISSER & $72 \times 46-\mathrm{L} 13\left(4,0^{\circ}\right.$ Lat $\times 5,0^{\circ}$ Lon $)-0,1 \mathrm{hPa}$ & Del Genio e Yao (1993) \\
\hline GFDL_CM2.0 & $144 \times 90-\mathrm{L} 24\left(2,0^{\circ}\right.$ Lat $\times 2,5^{\circ}$ Lon $)-2$ hpa & Moorthi e Suarez (1992) \\
\hline
\end{tabular}

antes do ano 2000, tem como base a concentração de gases de efeito estufa da ordem de 320 ppm de $\mathrm{CO}_{2}$.

\subsection{Definição do gradiente meridional de TSM no Atlântico Tropical}

Com os dados das anomalias de TSM, o gradiente meridional foi definido pela diferença entre as anomalias de TSM no setor norte do Atlântico tropical $\left(5^{\circ} \mathrm{N}-20^{\circ} \mathrm{N}\right.$ e $\left.60^{\circ} \mathrm{W}-30^{\circ} \mathrm{W}\right)$ e do setor sul do Atlântico Tropical $\left(5^{\circ} \mathrm{S}-20^{\circ} \mathrm{S}\right.$ e $30^{\circ} \mathrm{W}-10^{\circ} \mathrm{W}$ ) - Figura 1, entre fevereiro a maio e março a maio. Neste último período o gradiente meridional de TSM tem maior impacto na variabilidade da precipitação do setor norte do NEB (Nobre e Shukla, 1996), e o mesmo é climatologicamente o período mais chuvoso na região (Repelli e Alves, 1992).

\subsection{Métricas usadas no estudo}

As métricas usadas para comparar os dados observados e simulados foram: anomalias, o coeficiente de correlação de Pearson e o coeficiente de determinação (correlação ao quadrado). Grande parte das médias e anomalias calculadas mostradas nos resultados foram em função do período póssegunda guerra mundial (1950-1993), para quais as observações nos oceanos tiveram melhor cobertura espacial.

\section{RESULTADOS E DISCUSSÃO}

Antes de analisar o desempenho dos modelos na simulação do gradiente meridional de TSM no Atlântico Tropical, a Figura 2 mostra o desempenho dos modelos em simular a precipitação no setor norte do Nordeste. Observa-se, por essa figura, que todos os modelos conseguem reproduzir o ciclo sazonal da precipitação no setor norte do NEB, com o máximo de chuva centrado nos meses de março e abril, com declínio a partir de maio e um mínimo em setembro e outubro. Todos os modelos superestimam a precipitação nessa região, a exceção do modelo GFDL, que subestima a precipitação a partir do mês de maio. Nota-se também que todos os três modelos apresentam pouca diferença na chuva média mensal para as suas rodadas em análises. Com relação a subestimar ou superestimar a precipitação, essa característica depende do modelo, em geral das suas características físicas de como esses resolvem os principais processos físicos na atmosfera que estão diretamente ligados a produção de precipitação.

Marengo e Valverde (2007), analisando cinco modelos do IPCC-AR4 na América do Sul, mostraram que todos os modelos sazonalmente subestimam a chuva sobre a Amazônia, especificamente o GFDL e o GISS estendem a área de déficit para o Sudeste. Por outro lado, o HadCM3 subestimou a precipitação apenas sobre parte da Amazônia Brasileira e Guianas. Seus resultados também mostraram áreas com subestimativas sobre o sudeste, centro sul do Brasil, Bolívia e norte do Chile e Argentina ( $+4 \mathrm{~mm} / \mathrm{dia})$. Os cinco modelos superestimam as chuvas sobre os Andes Peruano-Bolivianos, sul do Chile e Argentina. Lin (2007), analisando 22 modelos do IPPC-AR4 mostrou que há um predomínio de superestimativa do total anual da precipitação na área tropical do globo terrestre, quando simuladas por esses modelos em relação às observações.

Em Silveira et al. (2012) foram feitas análises de 24 modelos do IPCC-AR4 e suas relações com a precipitação no Nordeste Setentrional. Seus resultados mostraram que, em média, os modelos na estação chuvosa (fevereiro a maio) superestimam a precipitação observada no Nordeste Setentrional. Com relação as avaliação dos modelos individualmente, o estudo citado, mostrou que o modelo Japonês MRI-CGCM2.3.2 foi o que melhor representou a variabilidade da precipitação nessa região, seguido pelos modelos BCCR-BCM2.0 (Norueguês) e CNRM-CM3 (Francês). Também nesses resultados de Silveira et al. (2012), os modelos analisados aqui tiveram a seguinte classificação no desempenho em simular a precipitação no Nordeste Setentrional: GISSE_ER (quarto lugar), IAP FGOALS1.0G (sexta lugar) e o GFDL2.0 ficou em décimo sexto lugar, entre os 25 modelos analisados.

A Figura 3 corrobora a análise da Figura 2, e mostra na variabilidade interanual que todos os modelos superestimam a precipitação no período de fevereiro a maio. Nota-se nessa figura que os modelos FGOALS1.0G e GFDL tiveram menor amplitude interanual. Uma possível explicação do porque a amplitude interanual do modelo GISSER ter tido uma similaridade mais próxima das observações, não simulada nos 

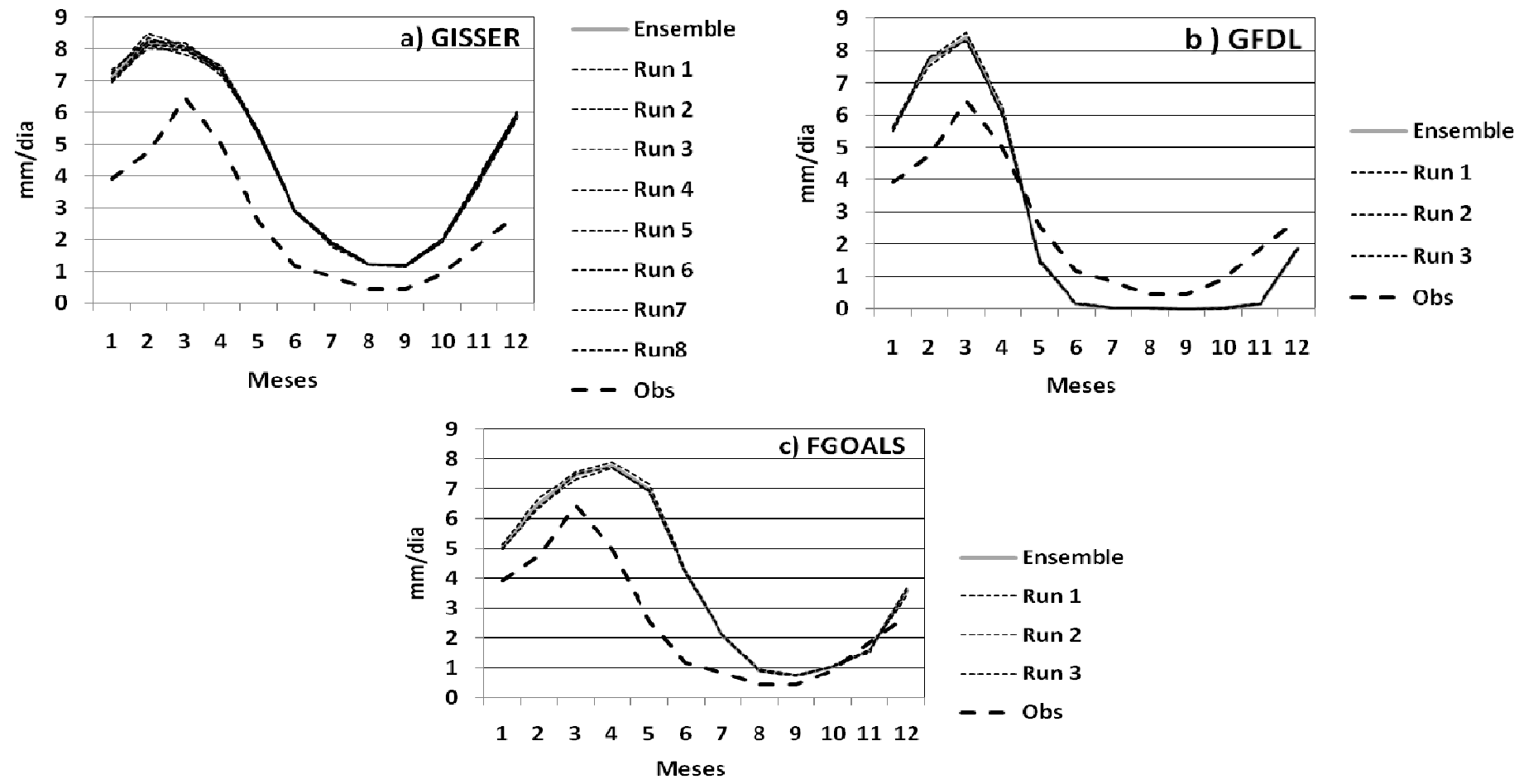

Figura 2 - Climatologia mensal da precipitação (mm/day) para 1901-1999 setor norte do NEB (45 ${ }^{\circ} \mathrm{W}-37^{\circ} \mathrm{W}$ e $\left.12^{\circ} \mathrm{S}-2^{\circ} \mathrm{S}\right)$. a) modelo GISSERxObs, b) moldeo GFDL_CM2.0xObs e c) Modelo FGOALS1.0GxObs. Obs significa precipitação obtida via dados East Anglia University.

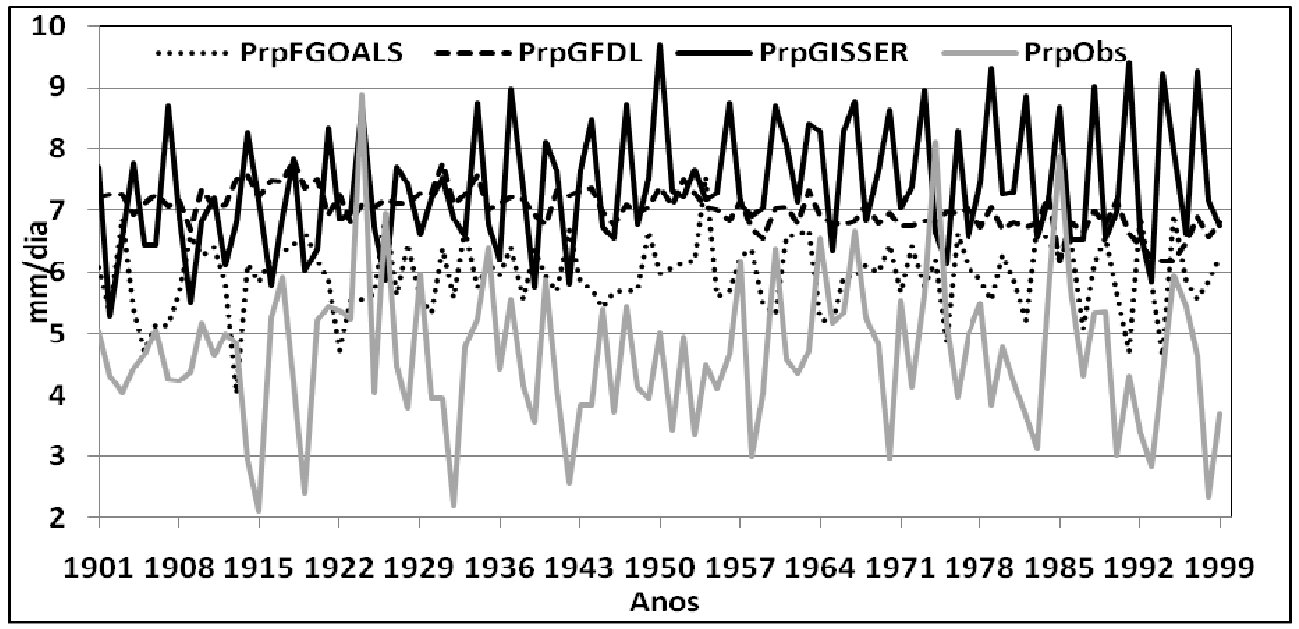

Figura 3 - Variabilidade interanual da precipitação (fevereiro a maio) no setor norte do NEB (1901-1999) observada e para a média das rodadas dos modelos GISSER, FGOALS1.0G e GFDL_CM2.0.

modelos FGOALS1.0G e GFDL, pode estar associada a uma melhor simulação do sinal interanual do gradiente meridional de TSM no Atlântico Tropical pelo modelo GISSER, acertando $37 \%$ dos anos com dipolos negativos e positivos, de $34 \%$ do modelo FGOALS1.0G e $32 \%$ do modelo GFDL, como pode ser visto na análise da Tabela 2 . Porém, vale ressaltar que a variabilidade pluviométrica interanual no setor norte do NEB, depende não somente da variabilidade térmica do Atlântico Tropical, mais também do que ocorre no Pacífico Tropical (Uvo et al. 1998).

A correlação embora não significativa estatisticamente a 95\% segundo o teste $\mathrm{t}$ Student (apenas valores em módulo acima 0,33 são significativos) mostrou que o modelo GISSER teve um marginal desempenho em acompanhar a variabilidade interanual 
da precipitação no setor norte do Nordeste do Brasil (GISSER $=0,16$, GFDL_CM2.0 $=-0,08$ e FGOALS1 $10 \mathrm{G}=-0,06$ ).

A Tabela 2 mostra a variabilidade interanual do gradiente meridional de TSM no Atlântico Tropical observado e simulado, para março a maio, pelos modelos acoplados e a precipitação média observada no setor norte do NEB e sua anomalia (1901-1999). Quando se compara a variabilidade das anomalias do gradiente meridional de TSM no Atlântico Tropical e precipitação, nota-se que não há uma relação direta de anomalias positivas (negativas) do gradiente meridional de TSM e a precipitação abaixo (acima) da média no setor norte do NEB. Porém, para alguns anos houve certo predomínio do gradiente meridional de TSM positivo, tanto o observado como o simulado, associados à precipitação observada abaixo da média consistente com as observações. Estes anos foram para o modelo GISSER $(1902,1904,1908,1911,1915,1937$, 1938, 1942, 1946, 1951, 1953, 1954, 1955, 1958, 1961, 1970, 1979, 1981, 1982, 1992 e 1997), para o modelo FGOALS1.0G (1902, 1903, 1904, 1908, 1909, 1911, 1915, 1919, 1930, 1931, 1936 e 1948) e para o GFDL $(1902,1903,1904,1907,1909$, 1911, 1936, 1938, 1941, 1942, 1946, 1951, 1954, 1955, 1956, 1958, 1961, 1962 e 1970).

Para os anos com gradiente meridional de TSM negativo observado e simulado, e precipitação observada acima da média os anos foram: modelo GISSER $(1913,1917,1921,1922,1923$, 1935, 1947, 1963, 1965, 1971, 1974, 1984, 1985, 1986, 1988 e 1996), para o modelo FGOALS1.0G (1923, 1924, 1934, 1935, 1937, 1947, 1950, 1957, 1964, 1965, 1971, 1973, 1974, 1975, 1984, 1985, 1986, 1988, 1989, 1994, 1995 e 1996) e para o modelo GFDL (1912, 1913, 1922, 1924, 1935, 1950, 1974, 1977, 1984, 1985, 1986, 1988 e 1995.

Percentualmente, o sucesso na simulação do sinal interanual do gradiente meridional de TSM positivo e precipitação observada abaixo da média entre 1901-1999 foi aproximadamente: Modelo GISSER (21\%), FGOALS1.0G (12\%) e GFDL (19\%). Por outro lado, o sucesso da simulação do sinal interanual do gradiente meridional de TSM negativo e precipitação observada acima da média entre 1901-1999 foi em torno de: Modelo GISSER (16\%), FGOALS1.0G (22\%) e GFDL (13\%).

Nota-se que os modelos GISSER e GFDL tiveram um percentual maior de anos corretos com o sinal do gradiente meridional de TSM observado e anomalia da precipitação observada quando este foi positivo, enquanto para o modelo FGOALS1.0G o maior número de acertos nesse sinal foi para os anos com o sinal negativo do gradiente meridional de TSM.

As correlações entre as anomalias de TSM observadas e simuladas nas áreas norte e sul do dipolo meridional de TSM para o período de fevereiro a maio (1901-1999) pelos modelos foram: área norte e sul: FGOALS1.0G 1.0G (-0,05 e +0,54), GISSER $(+0,30$ e $+0,55)$ e GFDL_CM2.0 $(+0,32$ e $+0,56)$.

A variação anual de calor latente (Tabela 3), médias mensais, mostra que os todos os modelos, comparado as observações, superestimam os valores do fluxo de calor latente em ambas as áreas do gradiente, com valores mais próximos em magnitude sendo simulados pelos modelos FGOALS1.0G e GFDL. Na diferença entre as duas áreas (área norte menos a área sul), também os modelos FGOALS1.0G e GFDL conseguiram capturar o maior fluxo de calor latente no setor sul da bacia a partir do mês de maio até agosto mostrado nos dados COADS.

Na Figura 4 são apresentadas médias (1950-1993) para março a maio da circulação atmosférica em 1000 mb, e a precipitação sobre o Atlântico Tropical das reanálises e para as simulações dos modelos GISSER, GFDL e FGOALS1.0G com objetivo de identificar que modelo melhor simula o padrão médio da circulação junto à superfície e intensidade, e localização da precipitação associada a ZCIT. Observa-se por essas figuras que a circulação no Atlântico Tropical foi melhor simulada pelos modelos GFDL e GISSER, enquanto o modelo FGOALS1.0G tem uma configuração no campo de ventos completamente diferente dos dados de reanálises. No campo de precipitação, os modelos GFDL e FGOALS1.0G tiveram a localização do centro de máxima precipitação, junto a costa da América do Sul, aproximada ao observado nas reanálises, porém subestimando a precipitação nessa área. $\mathrm{O}$ modelo GISSER colocou um máximo de precipitação ao norte da posição localizada nas reanálises.

A Figura 5 mostra as tendências decadais para fevereiro a maio (médias móveis centradas). Nota-se por essa figura, que os modelos tiveram um melhor desempenho na simulação da tendência positiva observada (1901-1999), porém não conseguiram reproduzir a variabilidade das tendências decadais e multidecadais dos dados observados. Exceto o modelo GFDL que conseguiu simular a fase positiva da oscilação multidecadal observada a partir de 1930. As correlações em alguns modelos em ambas as áreas explicaram mais de $50 \%$ e até $85 \%$ da variabilidade do gradiente observado, sendo a área sul que teve melhor simulação. Os coeficientes de correlação $(r)$ e de determinação $\left(r^{2}\right)$ entre as observações e as simulações para essa tendência decadal foram: área norte: FGOALS1.0G 1.0G ( $\left.\mathrm{r}=0,32 \mathrm{e}^{2}=0,10\right)$,

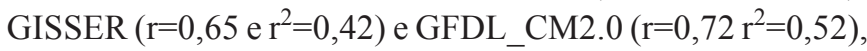

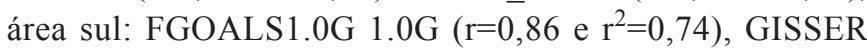
$\left(\mathrm{r}=0,93\right.$ e $\left.\mathrm{r}^{2}=0,86\right)$ e GFDL_CM2.0 $\left(\mathrm{r}=0,76 \mathrm{r}^{2}=0,58\right)$. Ressalta-se que correlações acima de 0,30 são significativas a $95 \%$ segundo teste t Student. Essa análise da tendência decadal, com médias móveis centradas, mostra um potencial de prognóstico futuros de médio prazos da tendência da 
Tabela 2 - Variabilidade interanual (1901-1999) do gradiente meridional de TSM (oC) observado e simulado e a precipitação observada e sua anomalia ( $\mathrm{mm} /$ dia) e sua média $(4,65 \mathrm{~mm} /$ dia) para o período de março a maio. Continua

\begin{tabular}{|c|c|c|c|c|c|c|c|}
\hline & GradObs & GradFGOALS1.0G & GradGISSER & GradGFDL & PrObs & MprObs & AnPrObs \\
\hline 1901 & 0,55 & 0,03 & 0,14 & 0,09 & 5,03 & 4,65 & 0,38 \\
\hline 1902 & 0,47 & 0,22 & 0,32 & 0,28 & 4,3 & 4,65 & $-0,35$ \\
\hline 1903 & 1,17 & 0,25 & $-0,4$ & 0,06 & 4,04 & 4,65 & $-0,61$ \\
\hline 1904 & 0,36 & 0,17 & 0,4 & 0,06 & 4,43 & 4,65 & $-0,22$ \\
\hline 1905 & 0,4 & 0,36 & $-0,28$ & 0,19 & 4,69 & 4,65 & 0,04 \\
\hline 1906 & 0,77 & $-0,09$ & 0,44 & 0,02 & 5,05 & 4,65 & 0,4 \\
\hline 1907 & 0,12 & $-0,16$ & $-0,27$ & 0,07 & 4,25 & 4,65 & $-0,4$ \\
\hline 1908 & 0,54 & 0,23 & 0,35 & $-0,03$ & 4,23 & 4,65 & $-0,42$ \\
\hline 1909 & 0,35 & 0,74 & $-0,15$ & 0,05 & 4,38 & 4,65 & $-0,27$ \\
\hline 1910 & $-0,32$ & 0,4 & 0,39 & 0,06 & 5,18 & 4,65 & 0,53 \\
\hline 1911 & 0,53 & 0,09 & 0,1 & 0,05 & 4,63 & 4,65 & $-0,02$ \\
\hline 1912 & $-0,6$ & 0,3 & 0,12 & $-0,07$ & 4,98 & 4,65 & 0,33 \\
\hline 1913 & $-0,81$ & 0,02 & $-0,2$ & $-0,18$ & 4,85 & 4,65 & 0,2 \\
\hline 1914 & $-0,04$ & $-0,14$ & $-0,04$ & $-0,21$ & 2,93 & 4,65 & $-1,72$ \\
\hline 1915 & 0,72 & 0,31 & 0,3 & $-0,15$ & 2,11 & 4,65 & $-2,54$ \\
\hline 1916 & $-0,16$ & 0,71 & 0,01 & 0,14 & 5,26 & 4,65 & 0,61 \\
\hline 1917 & $-0,58$ & 0,37 & $-0,19$ & 0,16 & 5,9 & 4,65 & 1,25 \\
\hline 1918 & $-0,29$ & 0,09 & $-0,2$ & 0,1 & 4,2 & 4,65 & $-0,45$ \\
\hline 1919 & 0,7 & 0,06 & $-0,03$ & $-0,08$ & 2,4 & 4,65 & $-2,25$ \\
\hline 1920 & $-0,61$ & 0,47 & 0,46 & 0,09 & 5,21 & 4,65 & 0,56 \\
\hline 1921 & $-0,66$ & 0,35 & $-0,19$ & 0,1 & 5,44 & 4,65 & 0,79 \\
\hline 1922 & $-0,44$ & 0,12 & $-0,01$ & $-0,01$ & 5,37 & 4,65 & 0,72 \\
\hline 1923 & $-0,24$ & $-0,17$ & $-0,04$ & 0,04 & 5,24 & 4,65 & 0,59 \\
\hline 1924 & $-0,57$ & $-0,34$ & 0,19 & $-0,06$ & 8,87 & 4,65 & 4,22 \\
\hline 1925 & $-0,64$ & $-0,12$ & 0,39 & 0,02 & 4,05 & 4,65 & $-0,6$ \\
\hline 1926 & $-0,26$ & 0,16 & 0,04 & 0,01 & 6,95 & 4,65 & 2,3 \\
\hline 1927 & 0,17 & $-0,03$ & 0,13 & 0,06 & 4,47 & 4,65 & $-0,18$ \\
\hline 1928 & $-0,16$ & $-0,11$ & $-0,12$ & 0,12 & 3,78 & 4,65 & $-0,87$ \\
\hline 1929 & 0,03 & 0,1 & 0,1 & 0,01 & 5,96 & 4,65 & 1,31 \\
\hline 1930 & 0,01 & 0,02 & $-0,16$ & $-0,13$ & 3,94 & 4,65 & $-0,71$ \\
\hline 1931 & 0,44 & 0,01 & $-0,07$ & $-0,09$ & 3,95 & 4,65 & $-0,7$ \\
\hline 1932 & 1,04 & $-0,09$ & 0,35 & $-0,14$ & 2,19 & 4,65 & $-2,46$ \\
\hline 1933 & 0,15 & $-0,25$ & $-0,2$ & $-0,07$ & 4,78 & 4,65 & 0,13 \\
\hline 1934 & $-0,77$ & $-0,08$ & 0,41 & $-0,23$ & 5,24 & 4,65 & 0,59 \\
\hline 1935 & $-0,32$ & $-0,11$ & $-0,36$ & $-0,02$ & 6,39 & 4,65 & 1,74 \\
\hline 1936 & 0,86 & 0,3 & $-0,08$ & 0,05 & 4,42 & 4,65 & $-0,23$ \\
\hline 1937 & $-0,22$ & $-0,28$ & 0,09 & 0,03 & 5,56 & 4,65 & 0,91 \\
\hline 1938 & 0,18 & $-0,22$ & 0,09 & 0,01 & 4,14 & 4,65 & $-0,51$ \\
\hline 1939 & $-0,33$ & 0,35 & $-0,01$ & $-0,08$ & 3,56 & 4,65 & $-1,09$ \\
\hline 1940 & 0,31 & $-0,07$ & $-0,37$ & 0,14 & 5,86 & 4,65 & 1,21 \\
\hline 1941 & 0,27 & $-0,11$ & $-0,13$ & 0,13 & 4,1 & 4,65 & $-0,55$ \\
\hline 1942 & 0,47 & $-0,12$ & 0,5 & 0,06 & 2,57 & 4,65 & $-2,08$ \\
\hline 1943 & 0,28 & $-0,26$ & $-0,34$ & $-0,07$ & 3,84 & 4,65 & $-0,81$ \\
\hline 1944 & $-0,13$ & $-0,43$ & 0,05 & $-0,15$ & 3,83 & 4,65 & $-0,82$ \\
\hline 1945 & 0,43 & $-0,28$ & 0,38 & $-0,12$ & 5,39 & 4,65 & 0,74 \\
\hline 1946 & 0,72 & $-0,16$ & 0,5 & 0,13 & 3,71 & 4,65 & $-0,94$ \\
\hline 1947 & $-0,3$ & $-0,29$ & $-0,14$ & 0,2 & 5,43 & 4,65 & 0,78 \\
\hline 1948 & 0,04 & 0,25 & $-0,31$ & $-0,11$ & 4,12 & 4,65 & $-0,53$ \\
\hline 1949 & $-0,51$ & $-0,15$ & $-0,01$ & $-0,14$ & 3,95 & 4,65 & $-0,7$ \\
\hline 1950 & $-0,15$ & $-0,35$ & 0,14 & $-0,08$ & 5,01 & 4,65 & 0,36 \\
\hline 1951 & 0,63 & $-0,56$ & 0,09 & 0,03 & 3,42 & 4,65 & $-1,23$ \\
\hline
\end{tabular}


Tabela 2 - Continuação

\begin{tabular}{|c|c|c|c|c|c|c|c|}
\hline 1952 & 0,33 & $-0,48$ & 0,1 & $-0,02$ & 4,94 & 4,65 & 0,29 \\
\hline 1953 & 0,32 & $-0,43$ & 0,69 & $-0,03$ & 3,35 & 4,65 & $-1,3$ \\
\hline 1954 & 0,38 & $-0,28$ & 0,48 & 0,02 & 4,5 & 4,65 & $-0,15$ \\
\hline 1955 & 0,19 & $-0,28$ & 0,05 & 0,08 & 4,11 & 4,65 & $-0,54$ \\
\hline 1956 & 0,45 & $-0,31$ & $-0,05$ & 0,04 & 4,62 & 4,65 & $-0,03$ \\
\hline 1957 & $-0,31$ & $-0,38$ & 0,16 & 0,11 & 6,17 & 4,65 & 1,52 \\
\hline 1958 & 1,54 & $-0,09$ & 0,19 & 0,07 & 3 & 4,65 & $-1,65$ \\
\hline 1959 & $-0,3$ & $-0,17$ & $-0,01$ & 0,01 & 4,03 & 4,65 & $-0,62$ \\
\hline 1960 & 0,1 & $-0,12$ & 0,03 & 0,04 & 6,38 & 4,65 & 1,73 \\
\hline 1961 & 0,19 & $-0,42$ & 0,2 & 0,01 & 4,58 & 4,65 & $-0,07$ \\
\hline 1962 & 0,29 & $-0,23$ & $-0,14$ & 0,07 & 4,35 & 4,65 & $-0,3$ \\
\hline 1963 & $-0,08$ & 0,11 & $-0,04$ & 0,27 & 4,72 & 4,65 & 0,07 \\
\hline 1964 & $-0,07$ & $-0,28$ & 0,37 & 0,01 & 6,55 & 4,65 & 1,9 \\
\hline 1965 & $-0,45$ & $-0,29$ & $-0,15$ & 0,15 & 5,18 & 4,65 & 0,53 \\
\hline 1966 & 0,35 & $-0,08$ & 0,26 & 0,03 & 5,34 & 4,65 & 0,69 \\
\hline 1967 & 0,04 & $-0,64$ & $-0,19$ & $-0,2$ & 6,66 & 4,65 & 2,01 \\
\hline 1968 & 0,03 & $-0,37$ & $-0,2$ & 0,09 & 5,26 & 4,65 & 0,61 \\
\hline 1969 & 0,46 & $-0,5$ & 0,21 & $-0,07$ & 4,82 & 4,65 & 0,17 \\
\hline 1970 & 0,8 & $-0,45$ & 0,13 & 0,06 & 2,95 & 4,65 & $-1,7$ \\
\hline 1971 & $-0,63$ & $-0,23$ & $-0,37$ & 0,02 & 5,53 & 4,65 & 0,88 \\
\hline 1972 & $-0,42$ & 0,01 & 0,31 & 0,05 & 4,13 & 4,65 & $-0,52$ \\
\hline 1973 & $-0,98$ & $-0,41$ & 0,02 & 0,07 & 5,65 & 4,65 & 1 \\
\hline 1974 & $-1,32$ & $-0,13$ & $-0,12$ & $-0,06$ & 8,13 & 4,65 & 3,48 \\
\hline 1975 & $-0,59$ & 0,2 & 0,3 & 0,08 & 5,16 & 4,65 & 0,51 \\
\hline 1976 & $-0,17$ & 0,07 & 0,11 & $-0,01$ & 3,96 & 4,65 & $-0,69$ \\
\hline 1977 & $-0,27$ & 0,08 & 0,19 & $-0,19$ & 4,98 & 4,65 & 0,33 \\
\hline 1978 & 0,65 & $-0,15$ & 0,34 & 0,05 & 5,49 & 4,65 & 0,84 \\
\hline 1979 & 0,28 & $-0,47$ & 0,07 & $-0,16$ & 3,83 & 4,65 & $-0,82$ \\
\hline 1980 & 0,38 & $-0,18$ & $-0,25$ & $-0,1$ & 4,78 & 4,65 & 0,13 \\
\hline 1981 & 0,87 & $-0,5$ & 0,29 & $-0,02$ & 4,2 & 4,65 & $-0,45$ \\
\hline 1982 & 0,18 & $-0,76$ & 0,12 & $-0,24$ & 3,66 & 4,65 & $-0,99$ \\
\hline 1983 & 0,6 & $-0,48$ & $-0,1$ & $-0,26$ & 3,12 & 4,65 & $-1,53$ \\
\hline 1984 & $-0,89$ & $-0,19$ & $-0,19$ & $-0,23$ & 5,66 & 4,65 & 1,01 \\
\hline 1985 & $-0,78$ & $-0,66$ & $-0,41$ & $-0,15$ & 7,89 & 4,65 & 3,24 \\
\hline 1986 & $-0,84$ & $-0,12$ & $-0,07$ & $-0,15$ & 5,71 & 4,65 & 1,06 \\
\hline 1987 & $-0,04$ & $-0,29$ & $-0,07$ & $-0,18$ & 4,31 & 4,65 & $-0,34$ \\
\hline 1988 & $-0,36$ & $-0,55$ & $-0,25$ & $-0,33$ & 5,33 & 4,65 & 0,68 \\
\hline 1989 & $-1,17$ & $-0,22$ & 0,5 & $-0,17$ & 5,36 & 4,65 & 0,71 \\
\hline 1990 & $-0,3$ & $-0,38$ & 0,15 & $-0,07$ & 3,01 & 4,65 & $-1,64$ \\
\hline 1991 & $-0,6$ & $-0,2$ & 0,17 & $-0,03$ & 4,31 & 4,65 & $-0,34$ \\
\hline 1992 & 0,1 & $-0,1$ & 0,09 & $-0,09$ & 3,39 & 4,65 & $-1,26$ \\
\hline 1993 & $-0,24$ & $-0,13$ & $-0,11$ & 0,01 & 2,84 & 4,65 & $-1,81$ \\
\hline 1994 & $-0,69$ & $-0,94$ & 0,09 & $-0,03$ & 4,33 & 4,65 & $-0,32$ \\
\hline 1995 & $-0,36$ & $-0,76$ & 0,28 & $-0,06$ & 5,94 & 4,65 & 1,29 \\
\hline 1996 & $-0,25$ & $-0,74$ & $-0,42$ & 0,05 & 5,44 & 4,65 & 0,79 \\
\hline 1997 & 0,68 & $-0,66$ & 0,22 & $-0,11$ & 4,63 & 4,65 & $-0,02$ \\
\hline 1998 & 0,02 & $-0,28$ & $-0,13$ & $-0,07$ & 2,34 & 4,65 & $-2,31$ \\
\hline 1999 & $-0,34$ & $-0,07$ & 0,43 & $-0,13$ & 3,69 & 4,65 & $-0,96$ \\
\hline
\end{tabular}


Tabela 3 - Médias mensais (1950-1993) do fluxo de calor latente $\left(\mathrm{W} / \mathrm{m}^{2}\right)$ nas áreas norte e sul, e suas diferenças (NA-SA), nas áreas do gradiente meridional de TSM no Atlântico Tropical.

\begin{tabular}{c|ccccccccccccc}
\hline \multicolumn{3}{c}{} & FGOALS & \multicolumn{4}{c}{ GFDL } & \multicolumn{3}{c}{ GISSER } & \multicolumn{3}{c}{ COADS } \\
\hline Meses & NA & SA & NA-SA & NA & SA & NA-SA & NA & SA & NA-SA & NA & SA & NA-SA \\
1 & 161,46 & 121,19 & 40,27 & 164,64 & 110,73 & 53,92 & 60,61 & 60,28 & 0,33 & 155,26 & 96,3 & 58,96 \\
2 & 161,34 & 124,39 & 36,95 & 159,75 & 120,78 & 38,97 & 61,87 & 59,79 & 2,08 & 152,54 & 100,38 & 52,17 \\
3 & 155,92 & 126,87 & 29,05 & 153,87 & 129,97 & 23,9 & 61,77 & 60,73 & 1,04 & 143,33 & 111,21 & 32,12 \\
4 & 145,85 & 131,89 & 13,96 & 148,11 & 143,62 & 4,49 & 61,87 & 60,45 & 1,42 & 133,89 & 122,53 & 11,36 \\
5 & 140,48 & 143,7 & $-3,22$ & 137,26 & 165,08 & $-27,82$ & 60,46 & 60,59 & $-0,13$ & 129,38 & 131,53 & $-2,15$ \\
6 & 138,15 & 166,76 & $-28,61$ & 115,56 & 172,52 & $-56,95$ & 60,28 & 59,08 & 1,2 & 123,9 & 138,32 & $-14,42$ \\
7 & 131,04 & 175,42 & $-44,38$ & 102,54 & 168,56 & $-66,02$ & 60,5 & 59,49 & 1,01 & 118,47 & 131,05 & $-12,57$ \\
8 & 120,02 & 168,94 & $-48,91$ & 106,71 & 150,68 & $-43,97$ & 61,24 & 61,49 & $-0,25$ & 116,46 & 117,24 & $-0,78$ \\
9 & 116,83 & 160,65 & $-43,81$ & 115,72 & 134,41 & $-18,69$ & 61,61 & 60,69 & 0,92 & 114,67 & 104,38 & 10,29 \\
10 & 126,15 & 143,82 & $-17,67$ & 123,39 & 119,85 & 3,54 & 61,78 & 59,54 & 2,23 & 121,85 & 98,69 & 23,16 \\
11 & 142,12 & 127,46 & 14,66 & 139,42 & 110,88 & 28,54 & 59,64 & 59,56 & 0,07 & 127,99 & 95,85 & 32,14 \\
12 & 153,16 & 118,16 & 35 & 158,51 & 106,65 & 51,87 & 61,37 & 61,07 & 0,31 & 146,29 & 94,76 & 51,53 \\
\hline
\end{tabular}

TSM nas áreas do gradiente meridonal no Atlântico Tropical com modelos acoplados oceano-atmosfera. Esses resultados de potencias prognósticos dessa tendência a médio prazo, diferem dos resultados mostrados em Meehl et al. (2007) e Latif (2006), que apresentaram uma perspectiva de prognósticos decadais em eventos oceâncos e atmósféricos no oceano Atlântico, como: Oscilação do Atlântico Norte e a Circulação Meridional de Retro-Alimentação (Meridional Overtunning Circulation), responsável pela transporte de densidade e massa entre os hemisférios no Oceano Atlântico.

A Figura 6 mostra a relação entre os índices de precipitação no setor norte do NEB e as anomalias de TSM nessa bacia ocêanica para as observações e simulada pelos modelos. O objetivo dessa análise foi identificar se a relação do padrão de correlação observado entre a precipitação no setor norte do NEB e as anomalias de TSM é reproduzido pelas simulações dos modelos. Isto é, um predomínio de correlações negativas (positivas) nos setores norte (sul) do Atlântico Tropical, que estão associadas a anomalias negativas (positivas) de precipitação no setor norte do NEB.

Essas análises se detiveram ao período de março a maio, pois é nesse trimestre que o impacto da variação de TSM no Atlântico Tropical é mais intenso na precipitação do setor norte do NEB (Nobre e Shukla, 1996). Nota-se por essa figura que nenhum modelo reproduz o padrão de correlação da Figura 6a. Os modelos GISSER e GFDL representam o sinal da correlação no setor sul da bacia, enquanto o FGOALS1.0G mostra uma inversão do padrão de correlação das observações, com configuração espacial não definida em ambas as bacias e com valores em magnitudes muito pequenos. A configuração dessas correlações simuladas pelos modelos só indicam que a variabilidade interanual da precipitação simulada pelos mesmos sobre o setor norte do NEB, diferentemente das observações, não parece ser controlada mais diretamente pela variação térmica do Atlântico Tropical. O que se deve, como visto na análise da Tabela 2, uma inconsistência na maioria dos anos na entre 1901-1999 simulação das características térmicas do gradiente meridional de TSM no Atlântico Tropical comparado as observações.

\section{SUMÁRIO E CONSIDERAÇÕES}

Este estudo mostra alguns resultados de simulações climáticas (1901-1999), com ênfase ao período de março a maio, de três modelos acoplados oceano-atmosfera do IPCC-AR4 do gradiente de TSM no Atlântico Tropical e sua relação com a precipitação no setor norte do Nordeste. Entre os principais resultados destaca-se que na variabilidade interanual (1901-1999) os modelos não tiveram bom desempenho em simular as fases e a amplitude do GradObs. Porém, a relação física do sinal do gradiente meridional de TSM no Atlântico Tropical com a precipitação abaixo ou acima da média do setor norte do NEB, período de março a maio foi consistente com as observações em alguns períodos de anos. Para os anos com sinal positivo no gradiente meridional de TSM observado e simulado com precipitação observada abaixo da média, o modelo GISSER reproduziu melhor essa relação com aproximadamente $21 \%$ de acerto dos anos. Quando o sinal desse gradiente meridional de TSM no Atlântico Tropical simulado e observado foi negativo e a precipitação observada foi acima da média, o modelo FGOALS1.0G teve melhor desempenho acertando em torno de $22 \%$ dos anos entre 1901-1999. Analisando-se as áreas isoladas do gradiente (norte e sul), as anomalias da tendência decadal da TSM dessa 
a) Reanalises (MAM)

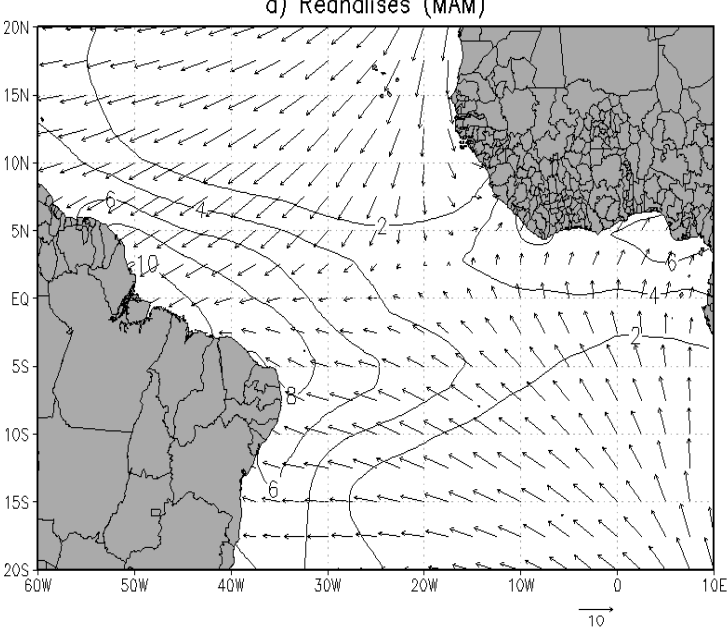

c) GFDL (MAM)

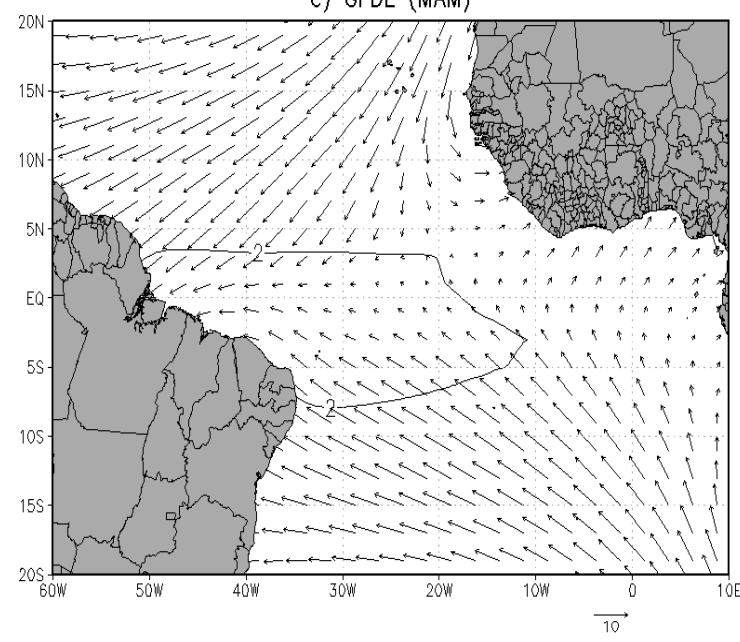

b) GISSER (MAM)

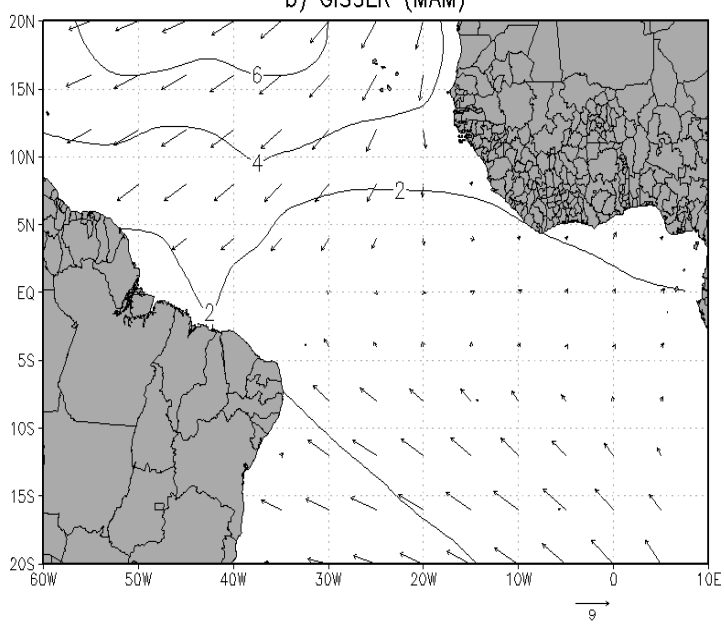

d) FGOALS (MAM)

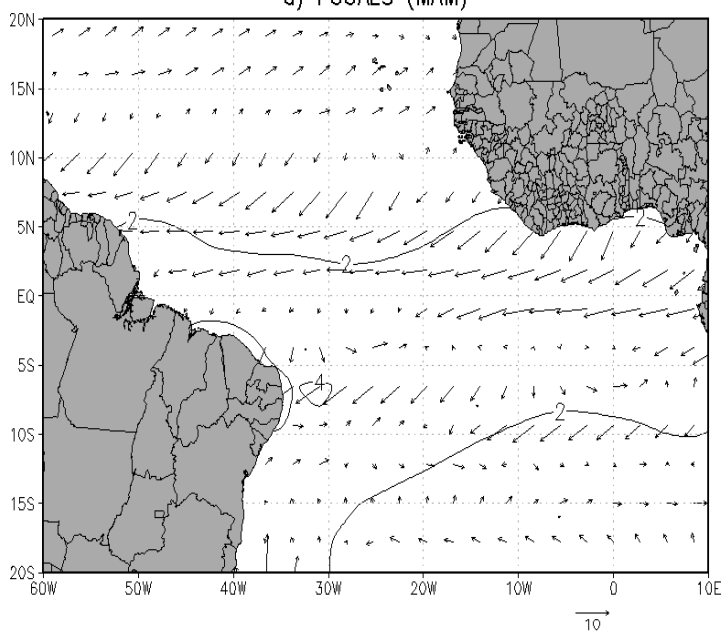

Figura 4 - Médias (1950-1993) de vento (m/s) em 1000 hPa e precipitação (mm/dia) no Atlântico Tropical. a) Reanálises, b) GISSER, c) GFDL e d) FGOALS1.0G. Para precipitação foram plotados valores maior ou igual a $2 \mathrm{~mm} / \mathrm{dia}$.

área sul foi melhor representada pelos modelos comparados aos dados de reanálises, implicando em uma boa simulação dessa área da bacia, com os modelos explicando mais de $60 \%$ e até mais de $80 \%$ da variabilidade dessa tendência decadal nessa área da bacia. A circulação atmosférica em $1000 \mathrm{hPa}$ e a precipitação associada a ZCIT no Atlântico Tropical, média para o período de março a maio, foi melhor simulada pelos modelos GISSER e GFDL, porém em ambos o máximo de precipitação junto a costa norte da América do Sul foi subestimada. O modelo GISSER embora tendo uma configuração de circulação similar as observações colocou o máximo de precipitação ao norte da posição climatológica. $\mathrm{O}$ modelo FGOALS1.0G simulou uma configuração de vento muito diferente das observações, embora tenha posicionado o máximo de precipitação junto à costa da América do Sul, mais com subestimativa em relação às reanálises. Para estudos futuros pretende-se analisar mas modelos acoplados do
IPCC-AR4 e IPCC-AR5, para identificar quais modelos tem melhor desempenho na simulação do gradiente meridional de TSM no Atlântico Tropical.

\section{AGRADECIMENTOS}

José Maria Brabo Alves, agradece a Coordenação de Aperfeiçoamento de Pessoal de Nível Superior (CAPES), pela bolsa de Pós-Doutorado Júnior (Processo No. 23038.007455/2011-95 PNPD CAPES-UFRN, como parte do Projeto Institucional do Programa de Pós-Doutorado 2011, sob coordenação da Dra. Rosane Rodrigues Chaves. Jacques Servain agradece o CNPq pela bolsa de Pesquisator Visitante Especial (PVE) (Processo CNPq 313559/2013-9) dentro do Programa "Ciência sem Fronteiras" Mudanças Climáticas no Atlântico Tropical (MUSCAT) associado no Programa INCTAmbtropic (Processo 565054/2012-4). 

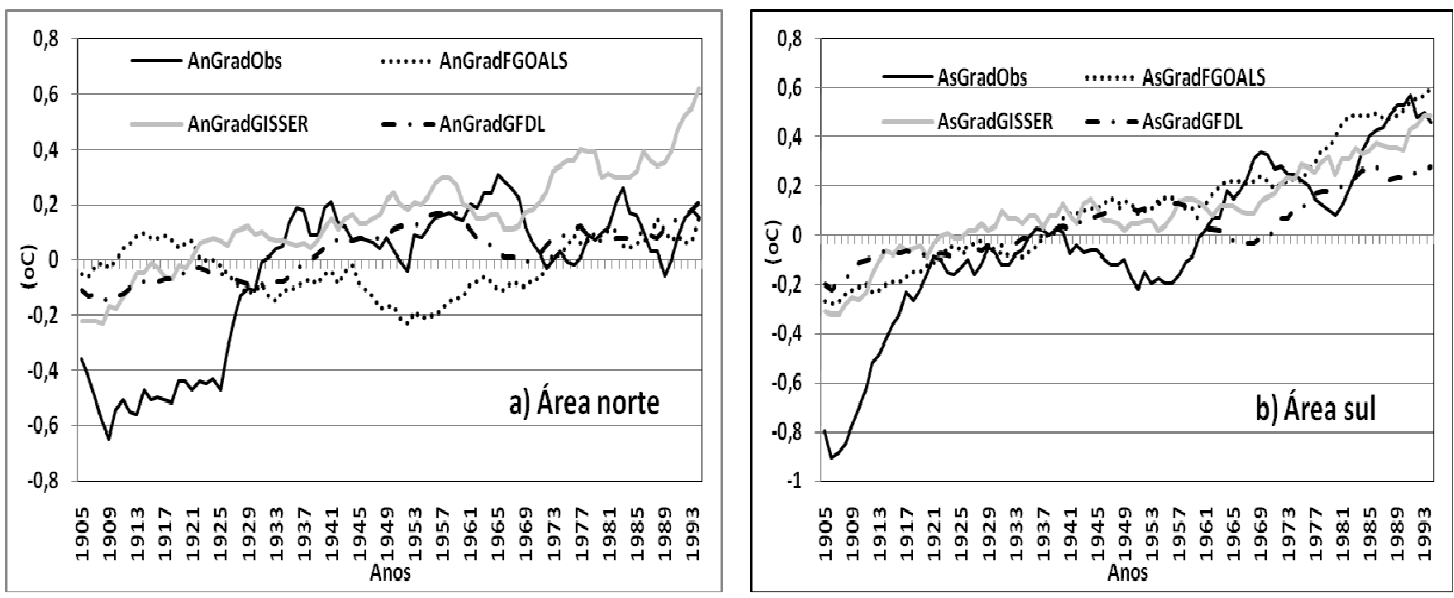

Figura 5 - Tendências (médias móveis decadais centradas para fevereiro a maio) entre 1901-1999, plotadas para os anos entre 1905-1994, observadas e simuladas (ver legendas) para as áreas norte e sul do gradiente meridional de TSM no Atlântico Tropical.

a) OBS $-r$ (TSM (MAM) $\times$ PRP (MAM)

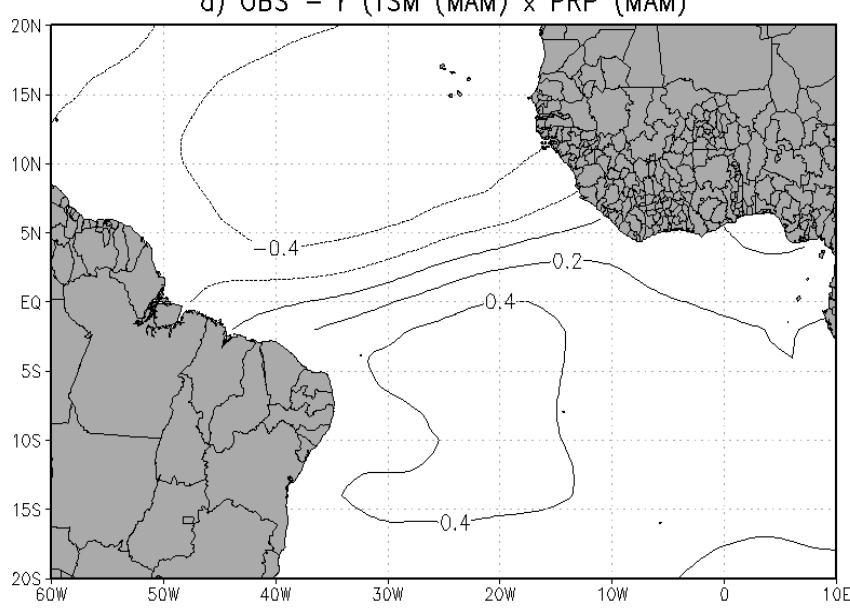

c) GFDL $-r($ TSM (MAM) $\times$ PRP (MAM)

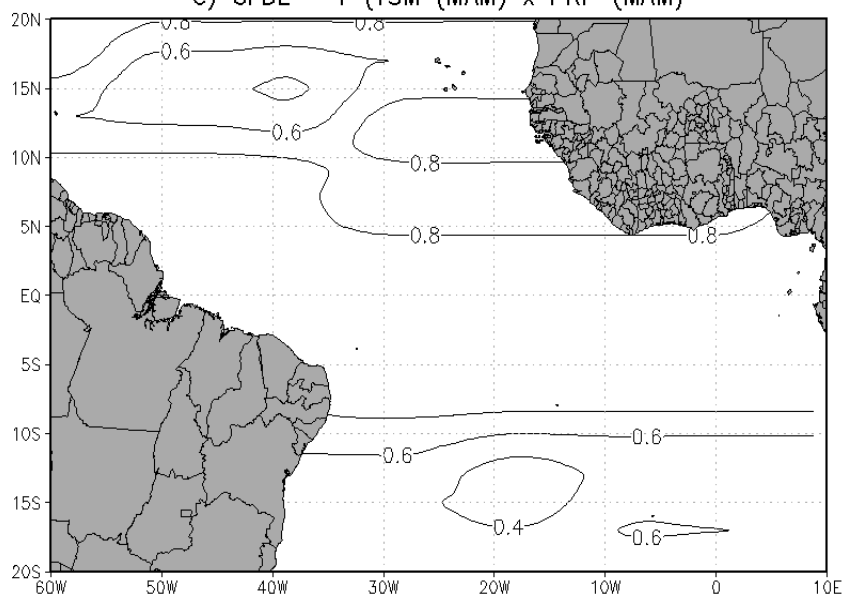

b) GISSER - $r$ (TSM (MAM) $\times$ PRP (MAM)

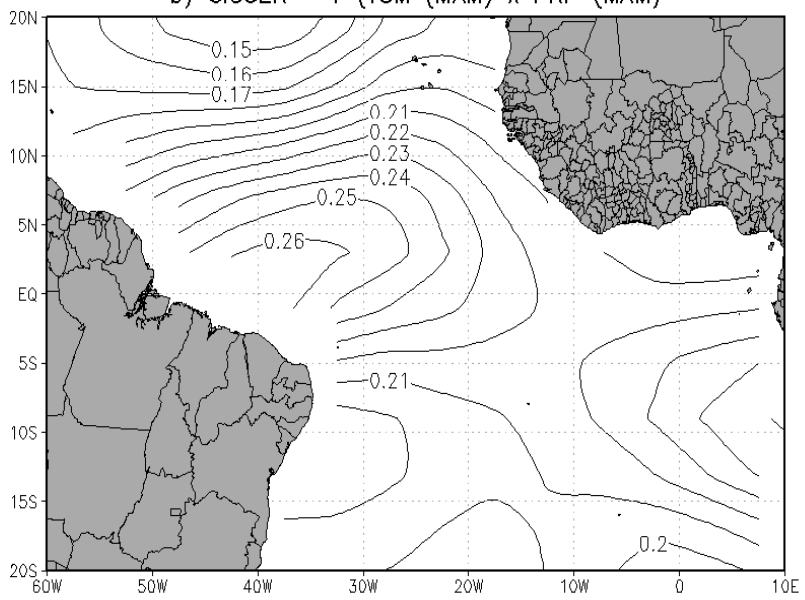

d) FGOALS $-r$ (TSM (MAM) $\times$ PRP (MAM))

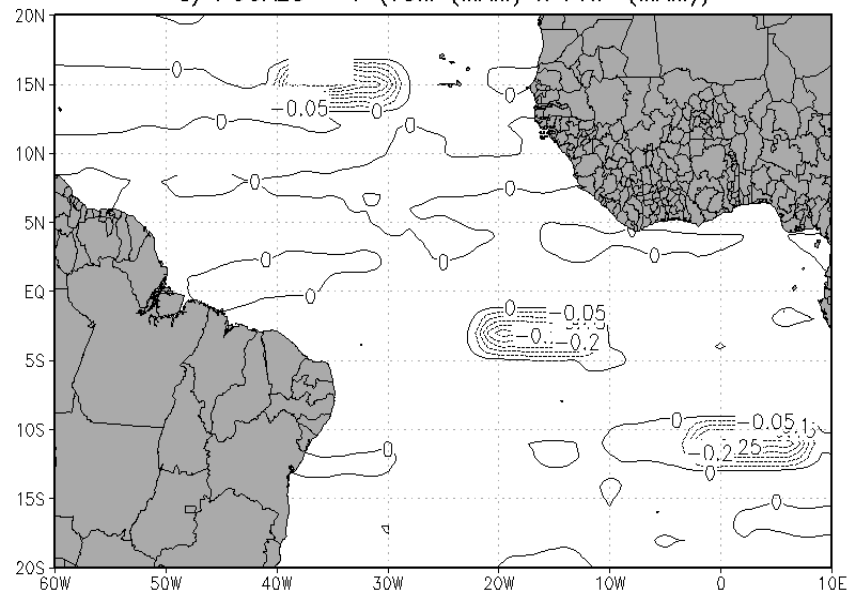

Figura 6 - Correlação linear (1950-1993), para o período de março a maio, entre os índices de precipitação no setor norte do NEB e do gradiente meridional de TSM no Atlântico Tropical observado e simulado pelo modelos. a) Reanálises, b) GISSER, c) GFDL e d) FGOALS1.0G. Correlações em módulo acima de 0,33 são significativas a 95\% segundo teste t Student. 


\section{REFERÊNCIAS BIBLIOGRÁFICAS}

BREUGEM, W. -P.; HAZELEGER, W.; HAASRMA, R. J. Multi-model study of tropical Atlantic variability and change. Geophysical Research Letter, DOI:10.1029. 2006.

DA SILVA, A, YOUNG, A. C, and LEVITUS, S. "Atlas of surface marine data 1994, volume 1.: Algorithms and procedures.," Tech. Rep. 6, U.S. Department of Commerce, NOAA, NESDIS, 1994.

DEL GENIO, A.D., and YAO, M.-S: Efficient cumulus parameterization for long-term climate studies: The GISS scheme. In The Representation of Cumulus Convection in Numerical Models, AMS Meteor. Monograph. K.A. Emanuel, and D.A. Raymond, Eds., vol. 24, no. 46. American Meteorological Society, 181-184, 1993.

GOOD, P.; LOWE, J. A.; ROWELL, D. P. Understanding in future projections for the tropical Atlantic: relationships with the unforced climate. Climate Dynamics, 32:203-218, DOI 10.1007/s00382-008-0466-7. 2009.

KALNAY, E.; KANAMITSU, M.; KISTLER, T.; COLLINS, W.; DEAVEN, D.; GANDIN, M.; IREDELL, M.; SHA, S.; WHITE, G.; WOOLEN, J.; ZHU, Y.; LEETMAA, A.; REYNOLDS, R.; CHELLIAH, M; EBISUZAKI, E.; HIGGINS, W.; JANOWIAK, J.; MO, K. C.; ROPELEWSKI, C.; WANG, J.; JENNE, R.; JOSEPH, D. The NCEP/ NCAR 40-Year Reanalysis Project. Bulletin of American Meteorological Society, v.77, p. 437-471. Doi: http://dx.doi. org/10.1175/1520-0477(1996)077<0437.TNYP>2.0.CO;2. 1996.

LATIF, M.; COLLINS, M.; POHLMANN, H.; KEENLYSIDE, N. A Review of Predictability Studies of Atlantic Sector Climate on Decadal Time Scales. Journal of Climate. 19., p.5971-5987. 2006.

LIN, J.-L. The double-ITCZ problem in IPCC AR4 coupled GCMs: Ocean-atmosphere feedback analysis. Journal of Climate, 20, p.4497-4525. 2007.

MARENGO, J. A., VALVERDE, M. C. Caracterização do clima no Século XX e Cenário de Mudanças de clima para o Brasil no Século XXI usando os modelos do IPCC-AR4. Revista Multiciência, Campinas, n. 8, p. 5-28, maio 2007.

MOORTHI, S., and SUAREZ, M. J. : Relaxed ArakawaSchubert: A parameterization of moist convection for general circulation models. Monthly Weater Review, 120, 978-1002, 1992.

MEEHL, G. A.; COVEY, C.; DELWORTH, T.; LATIF, M.; McAVANEY, B.; MIRCHELL, J. F. B.; STOUFFER, R. J.; TAYLOR, K. E. The WCRP CIMIP3 Multimodel Dataset. Anew Era in Climate Change Research. Bulletin of American Meteorological Society, v.9, p.1383-1394. 2007.
NEW, M. G., HULME, M, JONES, P. D. Representing twentieth century space-time climate variability. Part I: Development of a 1961-90 mean monthly terrestrial climatology. Journal of Climate, 12, p. 829-856. 1999.

NEW, M. G., HULME, M, JONES, P. D. Representing twentieth century spacetime climate variability. Part II: Development of 1901-96 monthly grids of terrestrial surface climate. Journal of Climate, 13, p. 2217-2238, 2000.

NOBRE, P.; SHUKLA, J. Variations of sea surface temperatures, wind stress, and rainfall over the tropical over the tropical Atlantic and South America. Journal of Climate, v.9, n.10, p.2464-2479. 1996.

REPELLI, C. A.; ALVES, J. M. B. A variabilidade pluviométrica no setor norte do Nordeste e os eventos El Niño-Oscilação Sul (ENOS). Revista Brasileira de Meteorologia, v. 7, n.2, p. 583-592, 1992.

SERVAIN, J.; LEGLER, D. M. Empirical orthogonal function analyses of tropical Atlantic

sea surface temperature and wind stress: 1964-1979. Journal of Geophysical Research, 91(C12): 14,181-14,191, 1986.

SILVEIRA, K. C.; SOUZA FILHO, F. A. DE.; LAZARÓ, Y. M. C.; COSTA, A. A. CASSAIN, D. S.; COUTINHO, M. M. Sazonalidade da Precipitação Sobre o Nordeste Setentrional Brasileiro nas Simulações do IPCC-AR4 . Revista Brasileira de Recursos Hídricos, v.17, n.3 - Jul/ Set, p.125-134. 2012.

SMITH, T. M., REYNOLDS, R.W., PETERSON, T. C., and LAWRIMORE, J.: Improvements NOAAs Historical Merged Land-Ocean Temp Analysis (1880-2006). Journal of Climate, v. 21, 2283-2296, 2008.

TANIMOTO, Y.; XIE, S. -P. Inter-hemispheric decadal variations in SST, wind surface, heat flux and cloud cover over the Atlantic Ocean. Journal of Meteorological of Society of Japan. 80, p.1199-1219. 2002.

UVO, C. B.; REPELLI, C. A.; ZEBIAK, S. E.; KUSHNIR, Y. The Relationships between Tropical Pacific and Atlantic SST and Northeast Brazil Monthly Precipitation. Journal of Climate, v. 11, p. 551-562, 1998.

ZHANG, G. J. e McFARLANE, N. A. M. Sensitivity of climate simulations to the parameterization of cumulus convection in the CCC-GCM. Atmosphere-Ocean, v.3, p.407-446. 1995.

ZEBIAK, E. S.Air-sea interactions in the equatorial Atlantic region. Journal of Climate, v.6, 8, P;15671568. doi:10.1175/15200442 (1993) 006<1567: AIITEA>2.0.CO;2. 